PROCEEDINGS OF THE

AMERICAN MATHEMATICAL SOCIETY

Volume 132, Number 9, Pages 2629-2638

S 0002-9939(04)07414-3

Article electronically published on April 8, 2004

\title{
SPECTRAL GAP FOR HYPERBOUNDED OPERATORS
}

\author{
FENG-YU WANG
}

(Communicated by Joseph A. Ball)

\begin{abstract}
Let $(E, \mathcal{F}, \mu)$ be a probability space, and $P$ a symmetric linear contraction operator on $L^{2}(\mu)$ with $P 1=1$ and $\|P\|_{L^{2}(\mu) \rightarrow L^{4}(\mu)}<\infty$. We prove that $\|P\|_{L^{2}(\mu) \rightarrow L^{4}(\mu)}^{4}<2$ is the optimal sufficient condition for $P$ to have a spectral gap. Moreover, the optimal sufficient conditions are obtained, respectively, for the defective log-Sobolev and for the defective Poincaré inequality to imply the existence of a spectral gap. Finally, we construct a symmetric, hyperbounded, ergodic contraction $C_{0}$-semigroup without a spectral gap.
\end{abstract}

\section{INTRODUCTION}

Let $(E, \mathcal{F}, \mu)$ be a probability space, and $P$ a symmetric contraction linear operator on $L^{2}(\mu)$ with $P 1=1$. Then the spectral radius of $P$ is $R(P)=1$. We say that $P$ has a spectral gap if

$$
R_{1}(P):=\sup \left\{\|P f\|_{L^{2}(\mu)}: \mu\left(f^{2}\right)=1, \mu(f)=0\right\}<1 .
$$

According to Simon and Høegh-Krohn [15], $P$ is called hyperbounded if

$$
\delta(P):=\|P\|_{L^{2}(\mu) \rightarrow L^{4}(\mu)}^{4}<\infty .
$$

We consider the following question raised in [15]: does a hyperbounded operator possess a spectral gap?

It turns out that the answer is NO for nonergodic positive definite operators. Recall that $P$ is called positive definite if $\mu(f P f) \geq 0$ for all $f \in L^{2}(\mu)$, and is called ergodic if (see [20])

$$
\lim _{n \rightarrow \infty} \frac{1}{n} \sum_{k=1}^{n} P^{k} f=\mu(f), \quad f \in L^{2}(\mu) ;
$$

here and in the sequel the limit is taken in $L^{2}(\mu)$. Let $\mathcal{H}:=\{f: P f=f\}$, and let $\pi: L^{2}(\mu) \rightarrow \mathcal{H}$ be the orthogonal projection. For positive definite $P$ it follows from the spectral theorem that

$$
\lim _{n \rightarrow \infty} P^{n} f=\pi f+\lim _{n \rightarrow \infty} \int_{0}^{1-} \lambda^{n} \mathrm{~d} E_{\lambda}(f)=\pi f, \quad f \in L^{2}(\mu),
$$

Received by the editors October 15, 2002 and, in revised form, June 3, 2003.

2000 Mathematics Subject Classification. Primary 47D07, 60H10.

Key words and phrases. Hyperboundedness, ergodicity, log-Sobolev inequality, spectral gap.

Supported in part by NNSFC(10025105, 10121101), TRAPOYT and the 973-Project. 
where $\left\{E_{\lambda}: \lambda \in[0,1]\right\}$ is the resolution of the identity of $P$. Therefore, it is easy to see that the ergodicity is equivalent to each of the following two statements (we write $f=g$ if these two functions are equal $\mu$-a.e.):

(a) for any $f \in L^{2}(\mu), P^{n} f \rightarrow \mu(f)$ as $n \rightarrow \infty$;

(b) for any $f \in L^{2}(\mu)$, if $P f=f$, then $f$ is constant.

Obviously, (a) and hence the ergodicity implies the positivity-improving property (or the $\mu$-essential irreducibility [11, 18]):

(c) for any $A, B$ with $\mu(A) \mu(B)>0$, there exists $n \geq 1$ such that $\mu\left(1_{A} P^{n} 1_{B}\right)>0$. Conversely, if $P$ is positive (or positivity-preserving), i.e., $P f \geq 0$ for all $f \geq 0$, then (c) implies (b) and hence is equivalent to the ergodicity. Indeed, for $P f=f$ with $\mu(f)=0$, one has $P f^{+} \geq(P f)^{+}=f^{+}$, where $f^{+}:=f \vee 0$. But $\mu\left(P f^{+}\right)=\mu\left(f^{+}\right)$, and so one has $P f^{+}=f^{+}$. Thus, for any $n \geq 1$ and any $\varepsilon>0$,

$$
0=\mu\left(f^{+} f^{-}\right)=\mu\left(f^{-} P^{n} f^{+}\right) \geq \varepsilon^{2} \mu\left(1_{\left\{f^{-}>\varepsilon\right\}} P^{n} 1_{\left\{f^{+}>\varepsilon\right\}}\right) .
$$

By (c) one has $\mu\left(f^{-}>\varepsilon\right) \mu\left(f^{+}>\varepsilon\right)=0$ for all $\varepsilon>0$. Hence, either $\mu\left(f^{+}\right)=0$ or $\mu\left(f^{-}\right)=0$. But $\mu(f)=0$ implies $\mu\left(f^{-}\right)=\mu\left(f^{+}\right)$, and one concludes that $f=0$. Therefore, (c) implies (b).

Let us recall some progress concerning this question. In the context of a symmetric contraction $C_{0}$-semigroup $\left(P_{t}\right)_{t \geq 0}$ with $P_{t} 1=1$, the Rothaus-Simon mass gap theorem says that the hypercontractivity (i.e., there exists $t>0$ such that $\left.\delta\left(P_{t}\right)=1\right)$ implies the existence of a spectral gap; see [13, 14] and also [8]. More precisely, if $\delta\left(P_{T}\right)=1$, then (see e.g. 4, Lemma 6.1.5])

$$
R_{1}\left(P_{t}\right) \leq 3^{-[t / T] / 2}, \quad t \geq T,
$$

where $[r]:=\sup \{n \in \mathbb{Z}: n \leq r\}$. According to [12, Theorem 2.3], this implies the following lower bound estimate for the spectral gap of the generator $(L, \mathcal{D}(L))$ :

$$
\operatorname{gap}(L):=\inf \left\{-\mu(f L f): f \in \mathcal{D}(L), \mu\left(f^{2}\right)=1, \mu(f)=0\right\} \geq \frac{\log 3}{2 T} .
$$

Therefore, $\delta\left(P_{T}\right)=1$ implies $R_{1}\left(P_{t}\right) \leq 3^{-t /(2 T)}$ for all $t \geq 0$.

Next, in 1998, Aida [1] proved that $\operatorname{gap}(L)>0$ provided there is $t>0$ such that $P_{t}$ is hyperbounded and is uniformly positivity-improving (Kusuoka [10]):

$$
\inf \left\{\mu\left(1_{A} P_{t} 1_{B}\right): \mu(A), \mu(B) \geq \varepsilon\right\}>0, \quad \varepsilon>0 .
$$

Obviously, the uniformly positivity-improving property is stronger than the ergodicity. In 2000, Wu [18 introduced the notion of uniform integrability for linear operators and studied the above question for ergodic, uniformly integrable, positive operators (see [18, Problem 3.10]). Moreover, Aida's above-mentioned result was generalized or extended in [9, 6] for general positive operators. Very recently, the essential spectral radius of Markov operators was estimated by $\mathrm{Wu}$ [19].

Our first observation in this note is that the hyperbound estimate $\delta(P)<2$ is the minimal sufficient condition for the existence of a spectral gap.

Theorem 1.1. (1) If $\delta(P)<2$, then $P$ has a spectral gap with

$$
R_{1}(P) \leq\left\{\frac{\sqrt{2 \delta(P)}}{3 \sqrt{2 \delta(P)}-4 \sqrt{\delta(P)-1}}\right\}^{1 / 2}<1 .
$$

(2) There exists a Markov operator $P$ with $\delta(P)=2$ and $R_{1}(P)=1$ (i.e., $P$ does not have a spectral gap). 
(3) If $P$ is positive definite and $\delta:=\delta(P)<2$, then $\left\|P^{r}\right\|_{L^{2}(\mu) \rightarrow L^{4}(\mu)}=1$ for all $r \geq r_{0}$, where

$$
r_{0}:=\frac{\log \{3+3(17 \delta-8-12 \sqrt{2 \delta(\delta-1)}) / 16\}}{\log (3-4 \sqrt{\delta-1} / \sqrt{2 \delta})}<\infty .
$$

Theorem 1.1 (1) generalizes 4, Lemma 5.5.11] where $\delta(P)=1$ is considered, and the proof of [4, Lemma 5.5.11] does not work for $\delta(P)>1$. Below is a direct consequence of Theorem 1.1 in the context of contraction $C_{0}$-semigroups, which improves the corresponding known result (see e.g. 4, Corollary 6.1.17]).

Corollary 1.2. Let $\left(P_{t}\right)_{t \geq 0}$ be the symmetric contraction $C_{0}$-semigroup generated by a negative definite selfadjoint operator $(L, \mathcal{D}(L))$ on $L^{2}(\mu)$ with $L 1=0$.

(1) If there exists $t>0$ such that $\delta\left(P_{t}\right)<2$, then

$$
\operatorname{gap}(L) \geq-\frac{1}{2 t} \log \frac{\sqrt{2 \delta\left(P_{t}\right)}}{3 \sqrt{2 \delta\left(P_{t}\right)}-4 \sqrt{\delta\left(P_{t}\right)-1}}>0 .
$$

(2) If $P_{t}$ is Markovian and the defective log-Sobolev inequality holds,

$$
\mu\left(f^{2} \log f^{2}\right) \leq-C_{1} \mu(f L f)+C_{2}, \quad f \in \mathcal{D}(L), \mu\left(f^{2}\right)=1,
$$

for some $C_{1}>0$ and $C_{2} \in[0, \log 2)$, then

$$
\operatorname{gap}(L) \geq-\frac{2}{C_{1} \log 3} \log \frac{\sqrt{2 \mathrm{e}^{C_{2}}}}{3 \sqrt{2 e^{C_{2}}}-4 \sqrt{\mathrm{e}^{C_{2}}-1}}>0 .
$$

In particular, if (1.4) holds for $C_{2}=0$, then $\operatorname{gap}(L) \geq \frac{2}{C_{1}}$, as is well known.

(3) There exists an example such that (1.4) holds for $C_{1}=0$ and $C_{2}=\log 2$, but $\operatorname{gap}(L)=0$.

Corollary 1.2 shows that $C_{2}<\log 2$ is the optimal sufficient condition for the defective $\log$-Sobolev inequality (1.4) to imply $\operatorname{gap}(L)>0$. Below we present the optimal sufficient condition for the defective Poincaré inequality to imply the existence of a spectral gap.

Theorem 1.3. Let $(L, \mathcal{D}(L))$ be the generator associated with a conservative Dirichlet form $(\mathcal{E}, \mathcal{D}(\mathcal{E}))$. If there exist $C_{1}>0$ and $C_{2} \in[1,2)$ such that

$$
\mu\left(f^{2}\right) \leq C_{1} \mathcal{E}(f, f)+C_{2} \mu(|f|)^{2}, \quad f \in \mathcal{D}(\mathcal{E}),
$$

then

$$
\operatorname{gap}(L) \geq \frac{1-\sqrt{C_{2}\left(C_{2}-1\right) / 2}}{2 C_{1}}>0 .
$$

On the other hand, there exists a conservative Dirichlet form such that (1.6) holds for $C_{2}=2$ but $\operatorname{gap}(L)=0$.

Finally, a counterexample is constructed in the paper to show that even in the ergodic case the hyperboundedness is insufficient for the existence of a spectral gap. This example enables us to state the following result.

Theorem 1.4. There exist $(E, \mathcal{F}, \mu)$ and a symmetric, hyperbounded, ergodic contraction $C_{0}$-semigroup $\left(P_{t}\right)_{t \geq 0}$ on $L^{2}(\mu)$ without a spectral gap. 
We remark that Theorem 1.4 does not provide any negative answer to Wu's problem 3.10 in [18, since the operator constructed in the counterexample is not positivity-preserving. Wu ([18, Example 1.8]) provided an example of a hyperbounded (but non- $C_{0}$ ) semigroup without a spectral gap.

\section{Proofs of Theorem 1.1 and Corollary 1.2}

Proof of Theorem 1.1. (1) For $\varepsilon \in(0,1)$ and $f \in L^{2}(\mu)$ with $\mu\left(f^{2}\right)=1$ and $\mu(f)=$ 0 , let $g:=\sqrt{\varepsilon}+\sqrt{1-\varepsilon} f$. We assume that $\mu\left((P f)^{3}\right) \geq 0$; otherwise simply replace $f$ by $-f$. Since $\mu(P f)=\mu(f P 1)=\mu(f)=0$ and since $\mu\left((P f)^{4}\right) \geq \mu\left((P f)^{2}\right)^{2}$, it follows that

$$
\begin{aligned}
\delta(P): & =\|P\|_{L^{2}(\mu) \rightarrow L^{4}(\mu)}^{4} \geq \mu\left((P g)^{4}\right) \\
= & \varepsilon^{2}+(1-\varepsilon)^{2} \mu\left((P f)^{4}\right)+6 \varepsilon(1-\varepsilon) \mu\left((P f)^{2}\right) \\
& \quad+4 \varepsilon^{3 / 2} \sqrt{1-\varepsilon} \mu(P f)+4 \sqrt{\varepsilon}(1-\varepsilon)^{3 / 2} \mu\left((P f)^{3}\right) \\
& \geq(1-\varepsilon)^{2} \mu\left((P f)^{2}\right)^{2}+6 \varepsilon(1-\varepsilon) \mu\left((P f)^{2}\right)+\varepsilon^{2} .
\end{aligned}
$$

Thus,

$$
\mu\left((P f)^{2}\right) \leq \frac{\sqrt{8 \varepsilon^{2}+\delta(P)}-3 \varepsilon}{1-\varepsilon}=: h(\varepsilon), \quad \mu(f)=0, \mu\left(f^{2}\right)=1, \varepsilon \in(0,1) .
$$

Therefore,

$$
R_{1}(P)^{2} \leq \xi(\delta(P)):=\inf _{\varepsilon \in(0,1)} h(\varepsilon) .
$$

It is easy to see that when $\delta(P)=1$ one has

$$
\xi(1)=\lim _{\varepsilon \uparrow 1} \frac{\sqrt{8 \varepsilon^{2}+1}-3 \varepsilon}{1-\varepsilon}=\frac{1}{3} .
$$

Next, for $1<\delta(P)<2$, the minimum of $h(\varepsilon)$ over $\varepsilon \in(0,1)$ is reached at

$$
\varepsilon(\delta(P)):=\delta(P)-\frac{3}{4} \sqrt{2 \delta(P)(\delta(P)-1)} .
$$

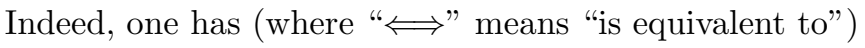

$$
\begin{aligned}
h^{\prime}(\varepsilon)=0 & \Longleftrightarrow(1-\varepsilon)\left(\frac{8 \varepsilon}{\sqrt{8 \varepsilon^{2}+\delta(P)}}-3\right)+\sqrt{8 \varepsilon^{2}+\delta(P)}-3 \varepsilon=0 \\
& \Longleftrightarrow 8 \varepsilon+\delta(P)=3 \sqrt{8 \varepsilon^{2}+\delta(P)} \\
& \Longleftrightarrow 8 \varepsilon^{2}-16 \delta(P) \varepsilon+9 \delta(P)-\delta(P)^{2}=0 \\
& \Longleftrightarrow \varepsilon=\delta(P)-\frac{1}{4} \sqrt{16 \delta(P)^{2}-2\left(9 \delta(P)-\delta(P)^{2}\right)}=: \varepsilon(\delta(P)) .
\end{aligned}
$$

Thus, $\xi(\delta(P))=h(\varepsilon(\delta(P)))$. It remains to calculate $h(\varepsilon(\delta))$ for $\delta \geq 1$. Observing that

$$
\begin{aligned}
& 8\left(\delta-\frac{3}{4} \sqrt{2 \delta(\delta-1)}\right)^{2}+\delta=8 \delta^{2}+9 \delta(\delta-1)-12 \delta \sqrt{2 \delta(\delta-1)}+\delta \\
& =8 \delta(\delta-1)-12 \delta \sqrt{2 \delta(\delta-1)}+9 \delta^{2}=(3 \delta-2 \sqrt{2 \delta(\delta-1)})^{2}
\end{aligned}
$$


and noting that $3 \delta>2 \sqrt{2 \delta(\delta-1)}$ for $\delta \geq 1$, one has

$$
\xi(\delta)=h(\varepsilon(\delta))=\frac{\sqrt{2 \delta(\delta-1)}}{3 \sqrt{2 \delta(\delta-1)}-4(\delta-1)}=\frac{\sqrt{2 \delta}}{3 \sqrt{2 \delta}-4 \sqrt{\delta-1}} .
$$

Therefore, (1.2) follows from (2.1).

(2) Let $E=\{0,1\}, \mu(0)=\mu(1)=\frac{1}{2}$ and $P=I$ (the identity operator). Then $P$ does not have any spectral gap. Next, let $f_{1}(0)=-1$ and $f_{1}(1)=1$. For any $f \in L^{2}(\mu)$ with $\mu\left(f^{2}\right)=1$, there exist $c_{1}, c_{2} \in \mathbb{R}$ such that $c_{1}^{2}+c_{2}^{2}=1$ and $f=c_{1}+c_{2} f_{1}$. Then

$$
\begin{aligned}
\mu\left((P f)^{4}\right) & =\mu\left(f^{4}\right)=c_{1}^{4}+c_{2}^{4}+6 c_{1}^{2} c_{2}^{2}=\left(c_{1}^{2}+c_{2}^{2}\right)^{2}+4 c_{1}^{2} c_{2}^{2} \\
& \leq \sup _{r \in(0,1)}\{1+4 r(1-r)\}=2,
\end{aligned}
$$

with equality holding when $c_{1}^{2}=c_{2}^{2}=\frac{1}{2}$. Thus, $\delta(P)=2$.

(3) For $f \in L^{2}(\mu)$ with $\mu\left(f^{2}\right)=1$, let $\hat{f}:=f-\mu(f)$. Let $R$ denote the upper bound of $R_{1}(P)$ given in (1), and simply denote $\delta=\delta(P)$. One has $R<1$, since $\delta<2$. Since $P$ is positive definite, $P^{r}$ is well-defined for $r \geq 1$ and we have $\mu\left(P^{r} \hat{f}\right)=\mu(\hat{f})=0$. Hence

$$
\mu\left(\left(P^{r} f\right)^{4}\right)=\mu(f)^{4}+4 \mu(f) \mu\left(\left(P^{r} \hat{f}\right)^{3}\right)+6 \mu(f)^{2} \mu\left(\left(P^{r} \hat{f}\right)^{2}\right)+\mu\left(\left(P^{r} \hat{f}\right)^{4}\right) .
$$

Noting that

$$
\begin{aligned}
& 4 \mu(f) \mu\left(\left(P^{r} \hat{f}\right)^{3}\right) \leq 4|\mu(f)| \sqrt{\mu\left(\left(P^{r} \hat{f}\right)^{4}\right) \mu\left(\left(P^{r} \hat{f}\right)^{2}\right)} \\
& \leq 2 t \mu(f)^{2} \mu\left(\left(P^{r} \hat{f}\right)^{2}\right)+2 t^{-1} \mu\left(\left(P^{r} \hat{f}\right)^{4}\right), \quad t>0, \\
& \mu\left(\left(P^{r} \hat{f}\right)^{2}\right) \leq R^{2 r} \mu\left(\hat{f}^{2}\right), \\
& \mu\left(\left(P^{r} \hat{f}\right)^{4}\right) \leq \delta \mu\left(\left(P^{r-1} \hat{f}\right)^{2}\right)^{2} \leq \delta R^{4(r-1)} \mu\left(\hat{f}^{2}\right)^{2},
\end{aligned}
$$

we obtain

$$
\mu\left(\left(P^{r} f\right)^{4}\right) \leq \mu(f)^{4}+2 R^{2 r}(3+t) \mu(f)^{2} \mu\left(\hat{f}^{2}\right)+(1+2 / t) \delta R^{4(r-1)} \mu\left(\hat{f}^{2}\right)^{2}, \quad t>0 .
$$

Letting

$$
t=\frac{1}{2}\left\{\sqrt{9+2 \delta R^{2 r-4}+\delta^{2} R^{4 r-8}}+\delta R^{2 r-4}-3\right\},
$$

we have

$$
\begin{aligned}
R^{2 r}(3+t) & =\delta R^{4(r-1)}(1+2 / t) \\
& =\frac{1}{2} R^{2 r}\left\{3+\delta R^{2 r-4}+\sqrt{9+2 \delta R^{2 r-4}+\delta^{2} R^{4 r-8}}\right\}=: h(r) .
\end{aligned}
$$

It suffices to prove that $h\left(r_{0}\right) \leq 1$. Indeed, if $h\left(r_{0}\right) \leq 1$, then it follows from (2.2) that

$$
\mu\left(\left(P^{r_{0}} f\right)^{4}\right) \leq\left\{\mu(f)^{2}+\mu\left(\hat{f}^{2}\right)\right\}^{2}=1
$$

for all $f \in L^{2}(\mu)$ with $\mu\left(f^{2}\right)=1$.

Let $s=3 \delta /\left(16 R^{4}\right)$; then $r_{0}=\frac{\log (3+s)}{\log R^{-2}}$ and

$$
3+2 s-\frac{\delta}{(3+s) R^{4}} \geq 3+\frac{3 \delta}{8 R^{4}}-\frac{\delta}{3 R^{4}}>0 .
$$


Moreover, $R^{2 r_{0}}=\frac{1}{3+s}$. Then

$$
\begin{aligned}
h\left(r_{0}\right) \leq 1 & \Longleftrightarrow \frac{1}{6+2 s}\left\{3+\frac{\delta R^{-4}}{3+s}+\left(9+\frac{2 \delta R^{-4}}{3+s}+\frac{\delta^{2} R^{-8}}{(3+s)^{2}}\right)^{1 / 2}\right\} \leq 1 \\
& \Longleftrightarrow\left(3+2 s-\frac{\delta R^{-4}}{3+s}\right)^{2} \geq 9+\frac{2 \delta R^{-4}}{3+s}+\frac{\delta^{2} R^{-8}}{(3+s)^{2}} \\
& \Longleftrightarrow 4 s^{2}+12 s \geq \frac{6 \delta R^{-4}(1+s)}{3+s} \\
& \Longleftrightarrow \frac{12 s^{2}+2 s^{3}+18 s}{1+s} \geq 3 \delta R^{-4}
\end{aligned}
$$

Since $s^{2} \geq 2 s-1$, we have

$$
12 s^{2}+2 s^{3}+18 s \geq 12 s^{2}+2 s(2 s-1)+18 s=16 s(1+s) .
$$

Therefore, $h\left(r_{0}\right) \leq 1$ follows from the fact that

$$
\frac{16 s(1+s)}{1+s}=16 s=3 \delta R^{-4}
$$

Proof of Corollary 1.2. (1) If $\delta\left(P_{t}\right)<2$ for some $t>0$, then by Theorem 1.1 one has

$$
\mu\left(\left(P_{t} f\right)^{2}\right) \leq \frac{\sqrt{2 \delta\left(P_{t}\right)}}{3 \sqrt{2 \delta\left(P_{t}\right)}-4 \sqrt{\delta\left(P_{t}\right)-1}}=: \varepsilon_{t}<1, \quad \mu(f)=0, \quad \mu\left(f^{2}\right)=1 .
$$

Thus, it follows from [16] Lemma 2.1] (see also [12, Lemma 2.2]) that

$$
\mu\left(\left(P_{s} f\right)^{2}\right) \leq \mu\left(\left(P_{t} f\right)^{2}\right)^{s / t} \mu\left(f^{2}\right)^{1-s / t} \leq \varepsilon_{t}^{s / t}, \quad s \in[0, t], \quad \mu(f)=0, \mu\left(f^{2}\right)=1 .
$$

Since all three terms in the above formula are equal when $s=0$, it follows that

$$
-2 \mu(f L f)=\left.\frac{\mathrm{d}}{\mathrm{d} s} \mu\left(\left(P_{s} f\right)^{2}\right)\right|_{s=0} \leq \frac{1}{t} \log \varepsilon_{t}, \quad f \in \mathcal{D}(L), \quad \mu(f)=0, \quad \mu\left(f^{2}\right)=1 .
$$

Thus, we arrive at the Poincaré inequality

$$
\mu\left(f^{2}\right)-\mu(f)^{2} \leq-\frac{2 t}{\log \varepsilon_{t}} \mu(f L f), \quad f \in \mathcal{D}(L),
$$

and hence (1.3) holds.

(2) If (1.4) holds, then by Gross' theorem one has $\left\|P_{t}\right\|_{L^{2}(\mu) \rightarrow L^{4}(\mu)} \leq \mathrm{e}^{C_{2} / 4}$ for $t:=\frac{C_{1}}{4} \log 3$, see e.g. 22, Theorem 1.5]. Hence (1.5) follows from (1.3). Finally, let $E=\{0,1\}$ with $\mu(0)=\mu(1)=\frac{1}{2}$. Let $L=0$ (hence the corresponding form $\mathcal{E}=0$ ), which does not have a spectral gap. We have, for any function $f$ with $\mu\left(f^{2}\right)=1$,

$$
\begin{aligned}
\mu\left(f^{2} \log f^{2}\right) & =\frac{1}{2}\left(f(0)^{2} \log f(0)^{2}+f(1)^{2} \log f(1)^{2}\right) \\
& \leq \frac{1}{2} \sup _{r \in[0,2]}\{r \log r+(2-r) \log (2-r)\}=\log 2 .
\end{aligned}
$$

Therefore, (1.4) holds for $C_{1}=0$ and $C_{2}=\log 2$. 


\section{Proof of Theorem 1.3}

To prove Theorem 1.3 we need the following lemma, which is a dual version of [17, Proposition 3.2] in the setting of infinite reference measures.

Lemma 3.1. Let $(L, \mathcal{D}(L))$ be associated with a Dirichlet form $(\mathcal{E}, \mathcal{D}(\mathcal{E}))$. Assume that there are four constants $\alpha_{1}, \alpha_{2}, \beta_{1}, \beta_{2}>0$ such that

$$
\mu\left(f^{2}\right) \leq \alpha_{1} \mathcal{E}(f, f)+\beta_{1}\left\{(\sup f)^{2} \wedge(\inf f)^{2}\right\}, \quad f \in \mathcal{D}(L), \mu(f)=0,
$$

and

$$
\mu\left(f^{2}\right) \leq \alpha_{2} \mathcal{E}(f, f)+\beta_{2} \mu(|f|)^{2}, \quad f \in \mathcal{D}(\mathcal{E}) .
$$

If $\beta_{1} \beta_{2}<2$, then

$$
\operatorname{gap}(L) \geq \frac{1-\sqrt{\beta_{1} \beta_{2} / 2}}{2\left(\alpha_{1} \vee \alpha_{2}\right)}>0 .
$$

Proof. Let $f \in \mathcal{D}(\mathcal{E})$ with $\mu(f)=0$ and $\mu\left(f^{2}\right)=1$. For any $r>0$, let $r^{\prime} \in[r, \infty]$ be such that either $\mu\left((f \wedge r) \vee\left(-r^{\prime}\right)\right)=0$ or $\mu\left(\left(f \wedge r^{\prime}\right) \vee(-r)\right)=0$. Indeed, if $\mu((f \wedge r) \vee(-r)) \geq 0$, then there exists $R^{\prime} \in[r, \infty]$ such that $\mu\left((f \wedge r) \vee\left(-r^{\prime}\right)\right)=0$ (note that $\mu(f \wedge r) \leq 0$ since $\mu(f)=0$, while if $\mu((f \wedge r) \vee(-r))<0$, then there exists $r^{\prime} \in[r, \infty]$ such that $\left.\mu\left(\left(f \wedge r^{\prime}\right) \vee(-r)\right)=0\right)$. Without loss of generality, assume that $\mu\left((f \wedge r) \vee\left(-r^{\prime}\right)\right)=0$ for some $r^{\prime} \geq r$. Let $f_{r}:=(f \wedge r) \vee\left(-r^{\prime}\right)$. It follows from (3.1) that

$$
\mu\left(f_{r}^{2}\right) \leq \alpha_{1} \mathcal{E}\left(f_{r}, f_{r}\right)+\beta_{1} r^{2}
$$

Next, one has

$$
\begin{aligned}
& \mu\left(\left|f-f_{r}\right|\right) \leq \mu\left((|f|-r)^{+}\right)=\mu\left((|f|-r) 1_{\{|f|>r\}}\right) \\
& \leq \sqrt{\mu(|f|>r)}-r \mu(|f|>r) \leq \sup _{s \in\left(0, r^{-1}\right)}\left(s-s^{2} r\right)=\frac{1}{4 r} .
\end{aligned}
$$

Moreover, one has $\mu\left(\left(f+r^{\prime}\right)^{-}\right)=\mu\left((f-r)^{+}\right)=\frac{1}{2} \mu\left(\left|f-f_{r}\right|\right)$, since $\mu\left(f-f_{r}\right)=0$. Then it follows from (3.2) and (3.4) that

$$
\begin{aligned}
& \mu\left(\left(f-f_{r}\right)^{2}\right)=\mu\left((f-r)^{+^{2}}\right)+\mu\left(\left(f+r^{\prime}\right)^{-2}\right) \\
& \quad \leq \alpha_{2}\left\{\mathcal{E}\left((f-r)^{+},(f-r)^{+}\right)+\mathcal{E}\left(\left(f+r^{\prime}\right)^{-},\left(f+r^{\prime}\right)^{-}\right)\right\}+\frac{\beta_{2}}{2} \mu\left(\left|f-f_{r}\right|\right)^{2} \\
& \quad \leq \alpha_{2}\left\{\mathcal{E}(f, f)-\mathcal{E}\left(f_{r}, f_{r}\right)\right\}+\frac{\beta_{2}}{32 r^{2}}
\end{aligned}
$$

where, in the last step, we have used the fact that (see e.g. [5, Lemma 2.2])

$$
\mathcal{E}\left(f_{r}, f_{r}\right)+\mathcal{E}\left((f-r)^{+},(f-r)^{+}\right)+\mathcal{E}\left(\left(f+r^{\prime}\right)^{-},\left(f+r^{\prime}\right)^{-}\right) \leq \mathcal{E}(f, f) .
$$

Combining (3.3), (3.4) and (3.5), we obtain

$$
\begin{aligned}
1 & =\mu\left(f^{2}\right) \leq 2 \mu\left(f_{r}^{2}\right)+2 \mu\left(\left(f-f_{r}\right)^{2}\right) \\
& \leq 2\left(\alpha_{1} \vee \alpha_{2}\right) \mathcal{E}(f, f)+2 \beta_{1} r^{2}+\frac{\beta_{2}}{16 r^{2}}
\end{aligned}
$$


Letting $r^{2}=\frac{1}{4} \sqrt{\beta_{2} /\left(2 \beta_{1}\right)}$, we arrive at

$$
\mathcal{E}(f, f) \geq \frac{1-\sqrt{\beta_{1} \beta_{2} / 2}}{2\left(\alpha_{1} \vee \alpha_{2}\right)},
$$

and hence the proof is finished.

Proof of Theorem 1.3. Let $f \in L^{2}(\mu)$ with $\mu(f)=0$. Applying (1.6) to $f-\inf f$, we obtain

$$
\mu\left(f^{2}\right) \leq C_{1} \mathcal{E}(f, f)+\left(C_{2}-1\right)(\inf f)^{2} .
$$

Similarly, the same holds for $(\sup f)^{2}$ in place of $(\inf f)^{2}$. Thus,

$$
\mu\left(f^{2}\right) \leq C_{1} \mathcal{E}(f, f)+\left(C_{2}-1\right)\left\{(\sup f)^{2} \wedge(\inf f)^{2}\right\}, \quad f \in \mathcal{D}(\mathcal{E}), \mu(f)=0 .
$$

Then the first assertion follows from Lemma 3.1

On the other hand, let $E=\{0,1\}$ with $\mu(0)=\mu(1)=\frac{1}{2}$. Let $L=0$ (hence the corresponding form $\mathcal{E}=0$ ), which does not have a spectral gap. We have, for any function $f$,

$$
\mu\left(f^{2}\right)=\frac{1}{2}\left\{f(0)^{2}+f(1)^{2}\right\} \leq \frac{1}{2}(|f(0)|+|f(1)|)^{2}=2 \mu(|f|)^{2} .
$$

Hence (1.6) holds for $C_{1}=0$ and $C_{2}=2$.

\section{A counterexample: Proof of Theorem 1.4}

Consider, for instance, $E_{0}:=\{0,1\}$ and

$$
L_{0} f(i):=\frac{1}{2}[f(j)-f(i)], \quad i, j \in E_{0}, j \neq i .
$$

Let $\mu_{0}(0)=\mu_{0}(1)=\frac{1}{2}$. Then $L_{0}$ is selfadjoint in $L^{2}\left(\mu_{0}\right)$ with spectrum $\{0,-1\}$, where the nontrivial eigenfunction is given by $u(0)=-1, u(1)=1$. Moreover, the corresponding Markov semigroup is determined by

$$
P_{t}^{(0)} f:=\mu(f)+\mathrm{e}^{-t} \mu(f u) u, \quad t \geq 0, f \in L^{2}\left(\mu_{0}\right) .
$$

Now, let $E=E_{0}^{\mathbb{N}}:=\left\{\left(x_{n}\right)_{n \geq 1}: x_{n} \in E_{0}, n \geq 1\right\}$ and let $\mu=\mu_{0}^{\mathbb{N}}$ be the product measure. Consider the semigroup

$$
P_{t}:=\prod_{n \geq 1} P_{t}^{(n)}, \quad t \geq 0
$$

where $P_{t}^{(n)}:=P_{t}^{(0)}$ acting on the $n$-th space. Therefore, $P_{t}$ is a symmetric $C_{0}$ Markov semigroup on $L^{2}(\mu)$ generated by

$$
L=\sum_{n=1}^{\infty} L_{0}^{(n)}
$$

with domain $\mathcal{D}(L)$ containing all functions $f \in L^{2}(\mu)$ such that $\sum_{n=1}^{\infty} L_{0}^{(n)} f$ converges in $L^{2}(\mu)$, where $L_{0}^{(n)}$ stands for the operator $L_{0}$ acting on the $n$-th variable. It is clear that the spectrum of $L$ contains merely eigenvalues $\{0,-1,-2, \cdots\}$, and the multiplicity of each eigenvalue is infinite. Let $\mathcal{H}_{i}$ denote the eigenspace w.r.t. the eigenvalue $-i$. One has $\mathcal{H}_{0}=\mathbb{R}$ and

$$
\mathcal{H}_{1}=\operatorname{span}\left\{f_{n}: f_{n}(x):=u\left(x_{n}\right), x \in E, n \geq 1\right\} .
$$


Let $\pi_{i}: L^{2}\left(\mu^{\mathbb{N}}\right) \rightarrow \mathcal{H}_{i}$ be the orthogonal projection, $i \geq 1$. Then the spectral representation gives

$$
L f=-\sum_{i=1}^{\infty} i \pi_{i}(f), \quad f \in \mathcal{D}(L) .
$$

We now define a new selfadjoint operator on $L^{2}(\mu)$ via the spectral representation

$$
\tilde{L} f:=-\sum_{i \neq 1} i \pi_{i}(f)-\sum_{n=1}^{\infty} \frac{1}{n} \mu\left(f f_{n}\right) f_{n}, \quad f \in \mathcal{D}(\tilde{L}),
$$

where $\mathcal{D}(\tilde{L})$ contains all $f \in L^{2}(\mu)$ such that the right-hand side of (4.1) makes sense in $L^{2}(\mu)$. Let $\tilde{P}_{t}$ be the corresponding $C_{0}$-semigroup. It is clear that

$$
\tilde{P}_{t} f=P_{t} f, \quad f \in \mathcal{H}_{1}^{\perp},
$$

where $\mathcal{H}_{1}^{\perp}$ denotes the orthogonal complement of $\mathcal{H}_{1}$.

Proposition 4.1. $\tilde{P}_{t}$ is a hyperbounded, ergodic, symmetric contraction $C_{0}$-semigroup without a spectral gap.

Proof. Since $L f_{n}=-\frac{1}{n} f_{n}, n \geq 1$, there is no spectral gap. Next, let $f \in L^{2}(\mu)$ be such that $\tilde{P}_{t} f=f$ for some $t>0$, and let $f^{\prime \prime}$ be the orthogonal projection of $f$ onto $\mathcal{H}_{1}^{\perp}$. We have $\sum_{n=1}^{\infty}\left(1-\mathrm{e}^{-t / n}\right) \mu\left(f f_{n}\right) f_{n}=0$, and hence $\mu\left(f f_{n}\right)=0$ for all $n \geq 1$. Thus, $f=f^{\prime \prime}$ and $P_{t} f=\tilde{P}_{t} f=f$. By the ergodicity of $P_{t}$, the function $f$ has to be constant. Therefore, $\tilde{P}_{t}$ is ergodic.

It remains to prove the hyperboundedness of $\tilde{P}_{t}$. For $f \in L^{2}(\mu)$ with $\mu\left(f^{2}\right) \leq 1$, one has

$$
f=f^{\prime}+f^{\prime \prime}=\sum_{n=1}^{\infty} c_{n} f_{n}+f^{\prime \prime}
$$

for some sequence $\left\{c_{n}\right\} \subset \mathbb{R}$ with $\sum_{n=0}^{\infty} c_{n}^{2}=\mu\left(f^{\prime 2}\right) \leq 1$, where $f^{\prime}$ and $f^{\prime \prime}$ denote, respectively, the orthogonal projections of $f$ onto $\mathcal{H}_{1}$ and $\mathcal{H}_{1}^{\perp}$. Since $\mu$ is the product measure and since $\mu\left(f_{n}\right)=\mu\left(f_{n}^{3}\right)=0$ and $f_{n}^{2}=f_{n}^{4}=1$ for all $n \geq 1$, it follows from Fatou's lemma that

$$
\begin{aligned}
\mu\left(\left(\tilde{P}_{t} f^{\prime}\right)^{4}\right) & \leq \liminf _{N \rightarrow \infty} \int_{E}\left(\sum_{n=1}^{N} c_{n} \mathrm{e}^{-t / n} f_{n}\right)^{4} \mathrm{~d} \mu \\
& \leq \sum_{n=1}^{\infty} c_{n}^{4}+\sum_{m, n=1}^{\infty} c_{m}^{2} c_{n}^{2} \leq 2 \mu\left(f^{\prime 2}\right)^{2} .
\end{aligned}
$$

On the other hand, since $P_{t}^{(0)}$ is hypercontractive, so is $P_{t}$ (see [7]). Hence there exists $t>0$ (independent of $f$ ) such that

$$
\mu\left(\left(\tilde{P}_{t} f^{\prime \prime}\right)^{4}\right)=\mu\left(\left(P_{t} f^{\prime \prime}\right)^{4}\right) \leq \mu\left(f^{\prime \prime 2}\right)^{2} .
$$

Combining this with (4.3), we arrive at

$$
\mu\left(\left(\tilde{P}_{t} f\right)^{4}\right) \leq 2^{3}\left\{\mu\left(\left(\tilde{P}_{t} f^{\prime}\right)^{4}\right)+\mu\left(\left(\tilde{P}_{t} f^{\prime \prime}\right)^{4}\right)\right\} \leq 2^{4}\left[\mu\left(f^{\prime 2}\right)+\mu\left(f^{\prime \prime 2}\right)\right]^{2}=2^{4} \mu\left(f^{2}\right)^{2} .
$$

Therefore, $\tilde{P}_{t}$ is hyperbounded.

\section{ACKNOWLEDGEMENT}

The author would like to thank Professor L. Wu for very useful communications and the referee for valuable comments. 


\section{REFERENCES}

[1] S. Aida, Uniform positivity improving property, Sobolev inequalities, and spectral gaps, J. Funct. Anal. 158 (1998), 152-185. MR 2000d:60125

[2] D. Bakry, On Sobolev and logarithmic Sobolev inequalities for Markov semigroups, in "New Trends in Stochastic Analysis" (K. D. Elworthy, S. Kusuoka and I. Shigekawa, eds.), pp. 43-75, World Scientific, 1997. MR 99m:60110

[3] E. B. Davies and B. Simon, Ultracontractivity and the heat kernel for Schrödinger operators and Dirichlet Laplacians, J. Funct. Anal. 59 (1984), 335-395. MR 86e:47054

[4] J. D. Deuschel and D. Stroock, Large Deviations, Pure and Appl. Math. Ser. 137, Academic Press, San Diego, 1989. MR 90h:60026

[5] F.-Z. Gong and F.-Y. Wang, Functional inequalities for uniformly integrable semigroups and application to essential spectrums, Forum Math. 14 (2002), 293-313. MR 2003a:47097]

[6] F. Gong and L. Wu, Spectral gap of positive operators and applications, C. R. Acad. Sci. Paris Sér. I Math. 331 (2000), 983-988. MR 2002a:47057

[7] L. Gross, Logarithmic Sobolev inequalities, Amer. J. Math. 97 (1975), 1061-1083. MR 54:8263

[8] L. Gross, Logarithmic Sobolev inequalities and contractivity properties of semigroups, Lecture Notes in Math., vol. 1563, 54-88, Springer-Verlag, Berlin, 1993. MR 95h:47061

[9] M. Hino, Exponential decay of positivity preserving semigroups on $L^{p}$, Osaka J. Math. 37 (2000), 603-624; 39 (2002), 771. MR 2001k:60109; MR 2003h:60112

[10] S. Kusuoka, Analysis on Wiener spaces II: differential forms, J. Funct. Anal. 103 (1992), 229-274. MR 93c:58230

[11] D. Revuz, Markov Chains, North-Holland, Amsterdam, 1976. MR 54:3852

[12] M. Röckner and F.-Y. Wang, Weak Poincaré inequalities and $L^{2}$-convergence rates of Markov semigroups, J. Funct. Anal. 185 (2001), 564-603. MR 2002j:47075

[13] O. S. Rothaus, Diffusion on compact Riemannian manifolds and logarithmic Sobolev inequalities, J. Funct. Anal. 42 (1981), 102-109. MR 83f:58080a

[14] B. Simon, A remark on Nelson's best hypercontractive estimates, Proc. Amer. Math. Soc. 55 (1976), 376-378. MR 53:4825

[15] B. Simon and R. Høegh-Krohn, Hypercontractive semigroups and two dimensional selfcoupled Bose fields, J. Funct. Anal. 9 (1972), 121-180. MR 45:2528

[16] F.-Y. Wang, Functional inequalities, semigroup properties and spectrum estimates, Infin. Dimen. Anal. Quantum Probab. Relat. Topics 3 (2000), 263-295. MR 2002b:47083

[17] F.-Y. Wang, Functional inequalities for the decay of sub-Markov semigroups, Potential Analysis 18 (2003), 1-23. MR 2004a:47051

[18] L. Wu, Uniformly integrable operators and large deviations for Markov processes, J. Funct. Anal. 172 (2000), 301-376. MR 2001e:60062

[19] L. Wu, Essential spectral radius for Markov semigroups (I): discrete time case, Probab. Theory Relat. Fields 128 (2004), 255-321.

[20] K. Yosida, Functional Analysis, sixth edition, Grundlehren der Mathematischen Wissenschaften, band 123, Springer-Verlag, Berlin, 1980. MR 82i:46002

Department of Mathematics, Beijing Normal University, Beijing 100875, People's RePublic of ChinA

E-mail address: wangfy@bnu.edu.cn 